\title{
Unmyelinated Nerve Fibers of the Human Mandibular Nerve
}

\author{
By
Naoko NONAKA, Noboru GOTO, Hiromitsu EZURE, Hiroyuki ISHIKAWA and Kazuhiko OKA

Department of Anatomy, Showa University School of Medicine

- Received for Publication, September 28, 2000

\begin{abstract}
Key Words: Mandibular nerve, Unmyelinated nerve fibers, Trigeminal nerve, Axon, Human
Summary: The aim of this research is to find and to evaluate morphometorically the unmyelinated nerve fibers in the human mandibular nerve using a light microscope. Our report demonstrates for the first time the presence of the unmyelinated nerve fibers of the human mandibular nerve stained by a special method. Our results also indicate that there is a morphometric change with aging in the unmyelinated axons of the nerve.
\end{abstract}

For a long time, the mandibular nerve, which is a branch of the trigeminal nerve, was believed to be composed essentially of myelinated nerve fibers. Although there have been several reports dealing with unmyelinated nerve fibers ${ }^{1,3-8}$, those concerning the trigeminal nerve are limited in numbers $^{1,8)}$. In general, there is a lack of sufficient data concerning the presence, numbers, distribution, sizes and functions of human unmyelinated nerve fibers. In the present study, we used a special staining method which enabled us to uncover the presence of unmyelinated nerve fibers in the human mandibular nerve and to conduct an evaluation that revealed morphometric changes during the aging process.

\section{Material and Methods}

Small sections of the mandibular nerve were removed from an area adjacent to the trigeminal ganglion from 6 human cadavers (4 males and 2 females) aged from 26 to 85 years (average age: 66.2 years). The causes of death indicated no direct or indirect connection with the nervous system. These were selected among 50 subjects under the condition that all teeth were present and normal. We consider all the branches of the trigeminal nerve to be normal. The methods employed in this study are same as in our previous researches ${ }^{3-5.7)}$ concerning the fixation, washing, dehydration, embedding, sectioning, morphometry and statistical analysis; the only difference concerved the staining method. The sections were stained by Goto's modification of Masson-Goldner's method. The shrinkage ratio of sections was the same as in our previous reports ( $10 \pm 0 \%$ in length). For more details, see the references ${ }^{2-5,7)}$.

\section{Results}

Microscopic findings of unmyelinated axons in the mandibular nerve will be explained regarding numbers, transverse areas and perimeters.

\section{Numbers of Unmyelinated Axons}

We counted the total number of unmyelinated axons within a unit area of $2192.5 \mu \mathrm{m}^{2}$ in the human mandibular nerve as being between 10 and 29 (average: $18.8 \pm 4.8$ ). The myelinated nerve fibers have black axons with surrounding red myelin sheaths (Fig. 1), while the unmyelinated nerve fiber is shown with a black or dark purple axon without a surrounding red myelin sheath (Fig. 1). The number of unmyelinated axons did not change with age $(\mathrm{r}=-0.0940)$.

Correspondence: Noboru Goto, M.D., Professor and Chairman, Department of Anatomy, Showa University School of Medicine, 5-8, Hatanodai 1, Shinagawa-ku, Tokyo 142-8555 


\section{Transverse Area of Unmyelinated Axons}

The transverse area of unmyelinated axons in the human mandibular nerve ranged from 0.77 to 1.71 (average: 1.37) $\mu \mathrm{m}^{2}$. Ascatter diagram with regression analysis between the average transverse area and age reveals that the average area of unmyelinated axons in the transverse section increased with age $(r=0.8564)$, and so did the total transverse area per unit area $(r=0.4847)$.

\section{Perimeter of Unmyelinated Axons}

The average perimeter of unmylinated axons in the human mandibular nerve was $4.13 \mu \mathrm{m}$. It, too, increased with age $(\mathrm{r}=0.9120)$. Furthermore, we also noticed a slight correlation between age and the total perimeter of unmyelinated axons $(r=0.2987)$

\section{Discussion}

The authors could not find any other reports in the literature regarding morphometric data on unmyelinated nerve fibers of the human mandibular nerve. As far as the methodology is concerned, fixation, embedding, staining and morphometry are the most important steps in this research. We tried several staining methods, such as LPH stain ${ }^{2)}$, Klüver-Barrera method, Mallory's azan stain, silver impregnations, and others. We concluded that Goto's modification of Masson-Goldner's method is the best one at present for the morphometric evaluation of unmyelinated nerve fibers. Thanks to this staining method, we can easily distinguish unmyelinated from myelinated axons. Moreover, it is also possible to count the numbers and to measure the transverse areas and perimeters of unmyelinated axons with the use of an imageanalyzer. Unmyelinated axons appeared dark blue, surrounded by a thin Schwann sheath staired in a light-blue color, whereas myelinated axons, stained in a color similar to that of unmyelinated axons, were surrounded by a thick myelin sheath stained in red. Regarding the size, unmyelinated axons were always smaller than myelinated axons. A regression analysis of unmyelinated axons disclosed a positive correlation between the tranverse area and age $(r=0.8564)$, which meant the axonal area increased especially after 60 years of age (Fig. 2). The regression analysis also revealed that there was a significant correlation between the perimeter of the axon and age $(r=0.9120)$ : it showed a definite increase of perimeters after 60 years of age (Fig. 3). There was, however, no correlation between the number of unmyelinated axons per unit area and age $(r=-0.0940)$. As one grows older, an unmyelinated axon might become larger, although the number of unmyelinated axon practically does not

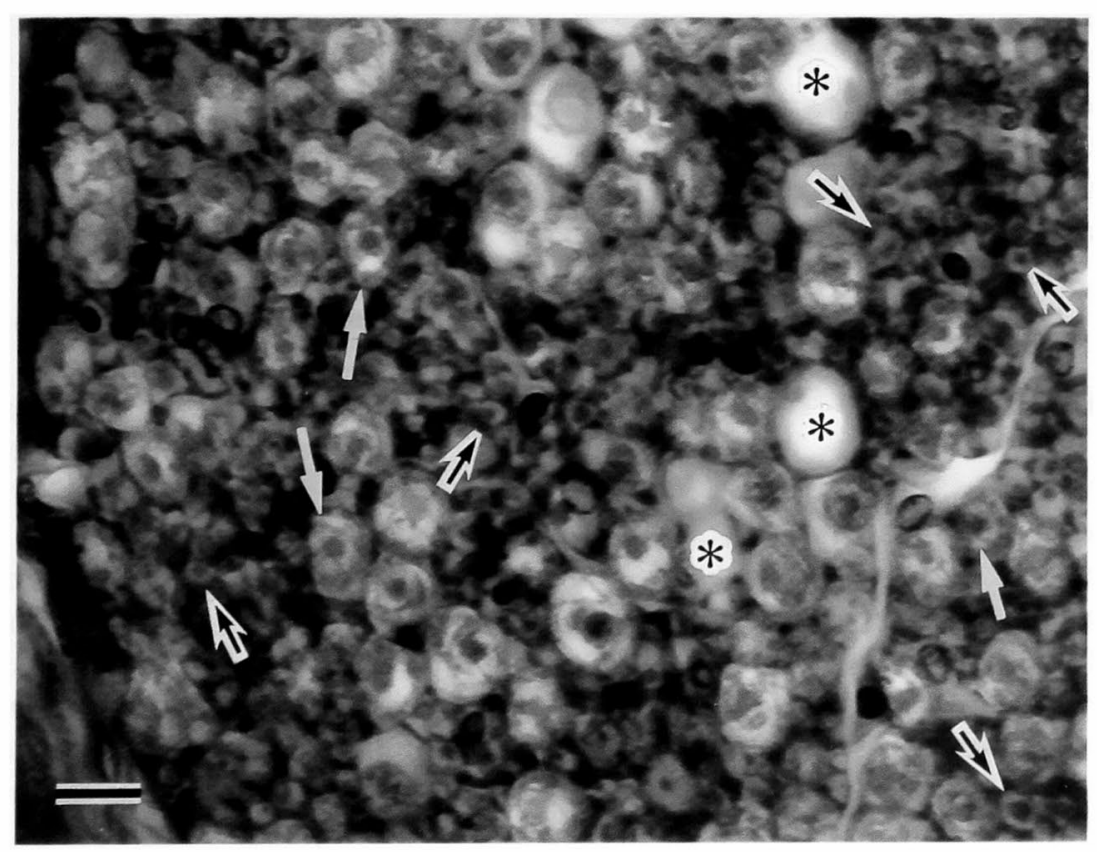

Fig. 1. Color microphoto of the mandibular nerve in 59-year-old man, Masson-Goldner's stain (Goto's modification).

There are many unmyelinated nerve fibers (black arrow) among myelinated nerve fibers (white arrow). *: degenerated nerve fibers, bar $=10 \mu \mathrm{m}$. 


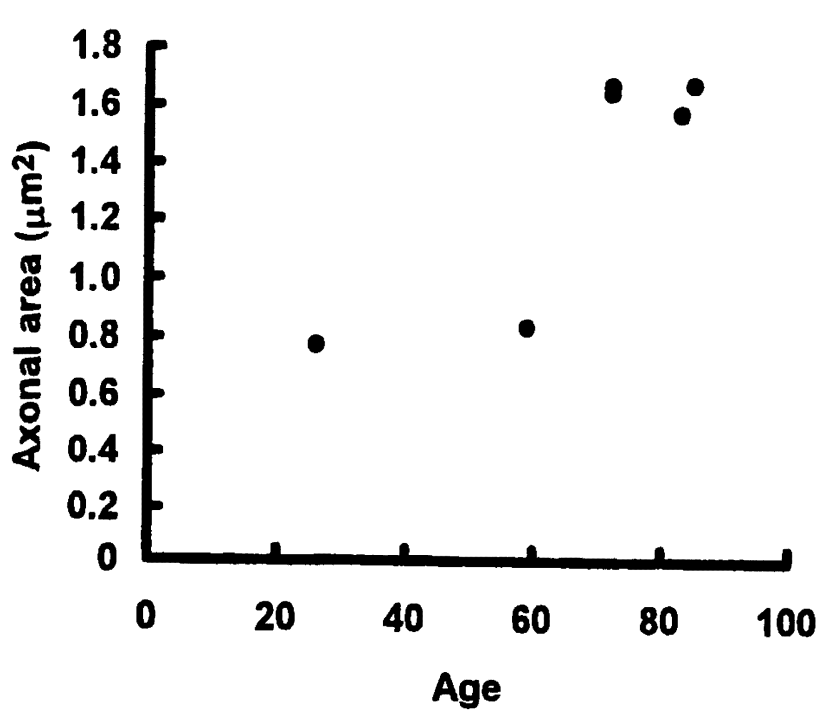

Fig. 2. Scatter diagram between the total transverse area of unmyelinated axons of the human mandibular nerve and age $(r=0.8564)$.

change $(r=-0.0940)$. Before this research, the presence of unmyelinated nerve fibers in human beings had not been established, nor had the changes occurring during the aging process. Researchers used to try to count only the number of myelinated nerve fibers in general. This research, however, has for the first time brough to light the increase of axonal sizes of unmyelinated nerve fibers during the aging process. The function of the unmyelinated nerve fibers in the human mandibular nerve still remains unknown.

\section{References}

1) Byers MR. Dental sensory receptor. Int Rev Neurobio 1984; 25:39-94.

2) Goto N. Discriminative staing methods for the nervous system: Luxol fast blue-periodic acid-Schiff-hematoxylin triple

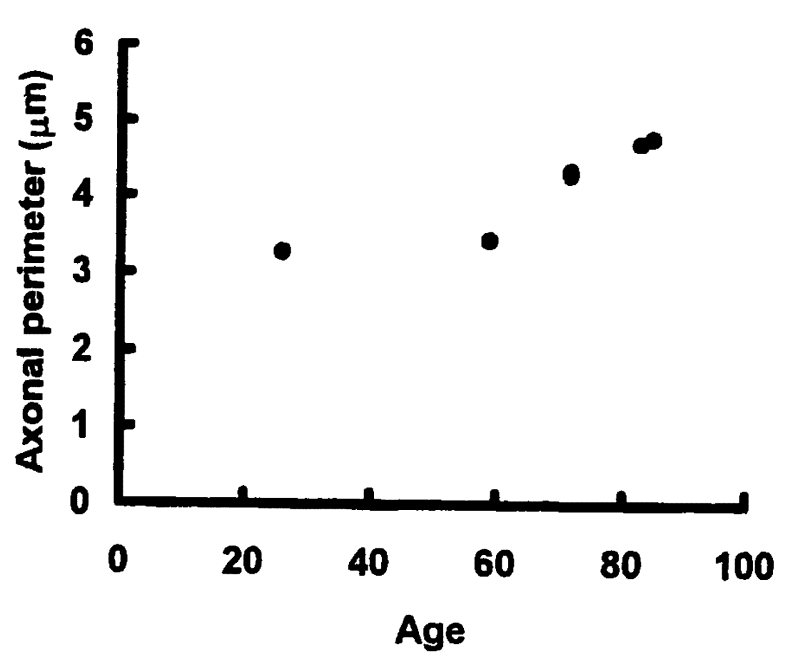

Fig. 3. Scatter diagram between the total perimeter of unmyelinated axons of the human mandibular nerve and age $(\mathrm{r}=0.9120)$.

stain and subsidiary staining methods. Stain Technol 1987; 62:305-315.

3) Kawamura N, Matsumoto K, Wada A, Goto N. Unmyelinated nerve fiber analysis of the human oculomoter nerve. Okajimas Folia Anat Jpn 2000; 77:59-62.

4) Kobayashi N, Sawabe Y, Matsumoto K, Otsuka N, Goto N. Unmyelinated nerve fiber analysis of the human abducent nerve. Okajimas Folia Anat Jpn 1998; 74:329-336.

5) Nagai Y, Goto N, Goto J, Kaneko Y, Suzuki H. Morphometric nerve fiber analysis and aging process of the human vestibular nerve. Okajimas Folia Anat Jap 1999; 76:95-100.

6) Pennisi E, Cruccu G, Manfredi M, Palladini G. Histometric study of myelinated fibers in the human trigeminal nerve. J Neurol Sci 1991; 105:22-28.

7) Suzuki K, Shiraishi N, Goto N, Suzuki M, Nonaka N. Unmyelinated nerve fiber analysis of the human lesser splanchnic nerve. Okajimas Folia Anat Jpn 2000; 76:285290.

8) Young RF, Stevens R. Unmyelinated axons in the trigeminal motor root of human and cat. J Comp Neurol 1979; 183:205-214. 